\title{
On Piterbarg Max-discretisation Theorem for Standardised Maximum of Stationary Gaussian Processes
}

\author{
Zhongquan $\operatorname{Tan}^{1}$ and Enkelejd Hashorva ${ }^{2}$
}

September 14, 2012

\begin{abstract}
With motivation from Hüsler (2004) and Piterbarg (2004) in this paper we derive the joint limiting distribution of standardised maximum of a continuous, stationary Gaussian process and the standardised maximum of this process sampled at discrete time points. We prove that these two random sequences are asymptotically complete dependent if the grid of the discrete time points is sufficiently dense, and asymptotically independent if the grid is sufficiently sparse. We show that our results are relevant for computational problems related to discrete time approximation of the continuous time maximum.
\end{abstract}

Key words: Extreme values; Piterbarg max-discretisation theorem; studentised maxima; Piterbarg inequality; approximation of random processes.

AMS Classification: Primary 60F05; secondary 60G15.

\section{Introduction and Main Result}

Let $\left\{X_{t}, t \geq 0\right\}$ be a standard (zero-mean, unit-variance) stationary Gaussian process (GP) with correlation function $r(t)$ and continuous sample paths. In this paper we assume that for some $\alpha \in(0,2]$ and $C>0$

$$
r(t)=1-C|t|^{\alpha}+o\left(|t|^{\alpha}\right) \text { as } t \rightarrow 0, \text { and } r(t)<1 \text { for } t>0 .
$$

If further the Berman condition

$$
\lim _{T \rightarrow \infty} r(T) \ln T=0
$$

holds, then Pickands theorem (see Pickands (1969) and Piterbarg (1972) for a rigorous proof of Pickands theorem) establishes the key limit result for the continuous time maximum $M_{T}=\max \left\{X_{t}, \forall t \in[0, T]\right\}$, namely

$$
\lim _{T \rightarrow \infty} \sup _{x \in \mathbb{R}}\left|P\left\{a_{T}\left(M_{T}-b_{T}\right) \leq x\right\}-\exp \left(-e^{-x}\right)\right|=0,
$$

where

$$
a_{T}=\sqrt{2 \ln T}, \quad b_{T}=a_{T}+a_{T}^{-1} \ln \left((2 \pi)^{-1 / 2} C^{1 / \alpha} H_{\alpha} a_{T}^{-1+2 / \alpha}\right) .
$$

Here $H_{\alpha} \in(0, \infty)$ denotes Pickands constant, which is defined as

$$
H_{\alpha}=\lim _{\lambda \rightarrow \infty} \frac{1}{\lambda} \mathbb{E}\left\{\exp \left(\max _{t \in[0, \lambda]} \sqrt{2} B_{\alpha / 2}(t)-t^{\alpha}\right)\right\}
$$

where $B_{\alpha}$ is a standard fractional Brownian motion with zero drift, continuous sample paths and $\mathbb{E}\left\{B_{\alpha}^{2}(t)\right\}=|t|^{2 \alpha}$, see e.g., Pickands (1969), Leadbetter et al. (1983), Lifshits (1995), Piterbarg (1996), Hashorva and Hüsler (2000), Dȩbicki (2002), Dȩbicki and Kisowski (2009), Falk et al (2010), Albin and Choi (2010), Dẹbicki and Tabiś (2011) for the basic properties and related constants. Note in passing that condition $r(t)<1$ for all $t>0$ is satisfied when Berman condition holds, see Leadbetter et. al (1983) p. 86.

\footnotetext{
${ }^{1}$ College of Mathematics, Physics and Information Engineering, Jiaxing University, Jiaxing 314001, PR China

${ }^{2}$ Department of Actuarial Science, Faculty of Business and Economics, University of Lausanne, Switzerland
} 
Our assumption on the $r(t)$, which is important for the study of studentized maximum is

$$
\lim _{T \rightarrow \infty} \frac{\ln T}{T} \int_{0}^{T}|r(t)-r(T)| d t=0 .
$$

An immediate consequence of (1),(4), spectral representation theorem and ergodic theorem is

$$
\lim _{T \rightarrow \infty} r(T)=\gamma \in[0,1)
$$

where $\gamma$ equals the atom at zero of the spectral distribution associated with $r(t)$, see McCormick (1980).

Set next

$$
\Delta_{T}:=\frac{M_{T}-\bar{X}_{T}}{\widetilde{r}_{T}}, \text { with } \widetilde{r}_{T}:=(1-r(T))^{1 / 2}, \quad \bar{X}_{T}:=\frac{1}{T} \int_{0}^{T} X_{t} d t
$$

and

$$
a_{T}:=\sqrt{2 \ln T}, \quad \beta_{T, \gamma}:=a_{T}+a_{T}^{-1} \ln \left((2 \pi)^{-1 / 2}\left(\frac{C}{1-\gamma}\right)^{1 / \alpha} H_{\alpha} a_{T}^{-1+2 / \alpha}\right) .
$$

For our contribution the following deep result of McCormick (1980) is crucial.

Theorem A. If $\left\{X_{t}, t \geq 0\right\}$ is a standard stationary GP with continuous sample paths and correlation function $r(t)$ satisfying (1) and (4), then we have

$$
\lim _{T \rightarrow \infty} \sup _{x \in \mathbb{R}}\left|P\left\{a_{T}\left(\Delta_{T}-\beta_{T, \gamma}\right) \leq x\right\}-\exp (-\exp (-x))\right|=0
$$

Several articles have been motivated by McCormick (1980); important asymptotic results for the standardised maximum $\Delta_{T}$ are derived in Ho and McCormick (1999), McCormick and Qi (2000) and James et al. (2007). For some earlier results see Berman (1962) and Grubbs (1964).

The interest for dealing with the problems presented above is not only theoretical; there are numerous applications where concrete computations are needed. Since observations cannot be made in continuous time, always in applications only the maximum taken at a discrete time grid can be calculated, and similarly for its standardised version $\Delta_{T}$. When the grid of sampling points becomes dense, the error of using a discrete process instead of the original continuous one decreases. Numerous theoretical results and applications can be found in the literature regarding the approximation of random processes, see e.g., the recent papers Abramowicz and Seleznjev (2011a,b,c), Hashorva et al. (2012) and the references therein. In the settings of this paper the situation is complicated due to the fact that rare events are involved. A pragmatical approach to approximate the distribution of the maximum $M_{T}$ or its standardised version is to run simulations. In order to run simulations, the time interval needs to be discretised, and the maximum on a discrete grid (see below) is then simulated. It is clear, that the continuous time maximum is not possible to simulate, and thus it remains to simulate the discretised version.

Now the question is if by simply running enough simulations it is possible to get accurate estimates of the probability of interest. We will answer this question in Section 3 where we present some possible applications of our theoretical results.

Piterbarg (2004) first studied the asymptotic relation between $M_{T}$ and the maximum of the discrete version

$$
M_{T}^{\delta}=\max \left\{X_{i \delta}, 0 \leq i \delta \leq T\right\}, \quad i \in \mathbb{Z}
$$

for some $\delta=\delta(T)>0$. Following Hüsler (2004) and Piterbarg (2004), a uniform grid $\mathfrak{R}=\mathfrak{R}(\delta)=\{k \delta: k=$ $0,1,2, \cdots\}$ with $\delta=\delta(T)>0$ is called sparse if

$$
\lim _{T \rightarrow \infty} \delta(T)(2 \ln T)^{1 / \alpha}=D,
$$


with $D=\infty$. If $D \in(0, \infty)$, the grid is referred to as a Pickands grid, and if $D=0$, the grid is called dense.

Under the above setting, the deep paper Piterbarg (2004) showed that the maximum $M_{T}^{\delta}$ taken over discrete time points and the maximum $M_{T}$ of the continuous time points can be asymptotically independent, dependent or totally dependent if the grid is a sparse, a Pickands or a dense grid, respectively. We refer to that result of Piterbarg (2004) as Piterbarg max-discretisation theorem.

Based on the findings of Hüsler (1990) Piterbarg max-discretisation theorem for a large class of locally stationary GP's introduced in Berman (1974) is proven in Hüsler (2004). Important related results for the storage process with fractional Brownian motion as input and the stationary non-Gaussian case can be found in Hüsler and Piterbarg (2004) and Turkman (2012), respectively. The recent contribution Tan and Wang (2012) presents Piterbarg maxdiscretisation theorem for strongly dependent stationary GP's.

With impetus from Hüsler (2004) and Piterbarg (2004) we derive in this paper Piterbarg max-discretisation theorem for standardised maximum of weakly dependent stationary GP's. Specifically, for $\Delta_{T}$ as in (6) and $\Delta_{T}^{\delta}$ defined by

$$
\Delta_{T}^{\delta}:=\frac{M_{T}^{\delta}-\bar{X}_{T}}{\widetilde{r}_{T}}
$$

we prove joint weak convergence, which is our main result presented below.

Theorem 1.1. Under the assumptions of Theorem A define for some $\delta=\delta(T)>0$

$$
\beta_{T}^{\delta}=a_{T}-a_{T}^{-1} \ln \left((2 \pi)^{1 / 2} \delta(T) a_{T}\right)
$$

and denote by $\mathfrak{R}(\delta)$ a sparse or a dense grid of points. Let further $a_{T}, \beta_{T, \gamma}$ be constants as defined in (7).

i) For any sparse grid $\mathfrak{R}(\delta)$

$$
\lim _{T \rightarrow \infty} \sup _{x \in \mathbb{R}, y \in \mathbb{R}}\left|P\left\{a_{T}\left(\Delta_{T}-\beta_{T, \gamma}\right) \leq x, a_{T}\left(\Delta_{T}^{\delta}-\beta_{T}^{\delta}\right) \leq y\right\}-\exp (-\exp (-x)-\exp (-y))\right|=0 .
$$

ii) If the grid $\mathfrak{R}(\delta)$ is dense, then

$$
\lim _{T \rightarrow \infty} \sup _{x \in \mathbb{R}, y \in \mathbb{R}}\left|P\left\{a_{T}\left(\Delta_{T}-\beta_{T, \gamma}\right) \leq x, a_{T}\left(\Delta_{T}^{\delta}-\beta_{T, \gamma}\right) \leq y\right\}-\exp (-\exp (-\min (x, y)))\right|=0 .
$$

The importance of Theorem 1.1 and of Piterbarg max-discretisation theorem in general is that apart from theoretical interest, it shows clearly for a given type of grid the right normalistions needed to achieve a good approximation. Using simply the same normalisation needed when continuous time standardised maximum is investigated is for sparse grids incorrect, see Section 3 below.

Organisation of the paper: In Section 2 we briefly discuss our findings and then present an implication of Theorem 1.1. Section 3 answers the question about validity of the simulation approach, and shows some ways how to deal with approximation error when utilising discrete grids. Based on our results, under a global Hölder condition (see (17) below) the mean error of the approximation tends to zero as the grid becomes dense. Additionally in that section we show that an appropriate choice of normalisation constants can be made by using the findings of this contribution. The proofs of our results are relegated to Section 4 which concludes this article.

\section{Discussion}

There is a close relation between the asymptotic behaviour of standardised maximum $\Delta_{T}$ and the studentised maximum

$$
\widetilde{\Delta_{T}}:=\frac{M_{T}-\bar{X}_{T}}{s_{T}},
$$


where $s_{T}^{2}:=\operatorname{Var}\left\{T \bar{X}_{T}\right\}$. Indeed, in view of McCormick (1980) and Ho and McCormick (1999) the result of Theorem A can be stated also for $\widetilde{\Delta_{T}}$. Our calculations show that it is however much more difficult to obtain similar results to Theorem 1.1 for the studentised maximum. Additionally, at present it seems also very difficult to obtain results for Pickands grids, which has been the case also in Hüsler (2004). Due to those difficulties the aforementioned cases shall be treated in a forthcoming research paper.

Our main findings in Theorem 1.1 show that the choice of the gird is very important for the asymptotic result.

A direct implication of Theorem 1.1 is that for any dense grid $\mathfrak{R}(\delta)$ we have

$$
a_{T}\left(\Delta_{T}-\Delta_{T}^{\delta}\right) \rightarrow 0, \quad T \rightarrow \infty
$$

in probability. The result in (12) is of interest for computational problems, and also simulations, see the discussion on the discretisation error in Section 3 below. Next, we formulate a direct consequence of Theorem 1.1, which is primarily of theoretical interest, and will be utilised in Section 3. Denote by $\Phi$ the distribution function of a standard Gaussian random variable.

Corollary 2.1. Under the Assumptions of Theorem A, if further $(r(T) \ln T)^{-1}=o(1)$, then for any sparse grid or any dense grid $\mathfrak{R}(\delta)$

$$
\lim _{T \rightarrow \infty} \sup _{x \in \mathbb{R}, y \in \mathbb{R}}\left|P\left\{r^{-1 / 2}(T)\left(\frac{M_{T}}{\widetilde{r}_{T}}-\beta_{T, \gamma}\right) \leq x, r^{-1 / 2}(T)\left(\frac{M_{T}^{\delta}}{\widetilde{r}_{T}}-\beta_{T}^{*}\right) \leq y\right\}-\Phi(\min (x, y) \sqrt{1-\gamma})\right|=0,
$$

where $\widetilde{r}_{T}:=(1-r(T))^{1 / 2}$ and $\beta_{T}^{*}=\beta_{T}^{\delta}$ for a sparse grid, $\beta_{T}^{*}=\beta_{T, \gamma}$ for a dense grid.

In the case that (5) holds with $\gamma=0$, i.e., $\lim _{T \rightarrow \infty} r(T)=\gamma=0$, then (13) leads to an extension of the main result of Mittal and Ylvisaker (1975) given in our final result below.

Corollary 2.2. Under the Assumptions of Theorem A, if further $\gamma=0$ and $(r(T) \ln T)^{-1}=o(1)$, then for any sparse grid or dense grid $\mathfrak{R}(\delta)$

$$
\lim _{T \rightarrow \infty} \sup _{x \in \mathbb{R}, y \in \mathbb{R}}\left|P\left\{\frac{M_{T}-\widetilde{r}_{T} \beta_{T, 0}}{r^{1 / 2}(T)} \leq x, \frac{M_{T}^{\delta}-\widetilde{r}_{T} \beta_{T}^{*}}{r^{1 / 2}(T)} \leq y\right\}-\Phi(\min (x, y))\right|=0,
$$

where $\beta_{T}^{*}=\beta_{T}^{\delta}$ for a sparse grid and $\beta_{T}^{*}=\beta_{T, 0}$ for a dense grid.

Note that if $\mathfrak{R}(\delta)$ is a dense grid, then (14) implies a similar result to (12), namely

$$
\frac{M_{T}-M_{T}^{\delta}}{r^{1 / 2}(T)} \rightarrow 0, \quad T \rightarrow \infty
$$

holds in probability.

To this end, we mention that for both standardised and studentised processes, it is of interest to consider maximum of those processes as in Kabluchko (2011) and Oesting et al. (2012).

\section{Discretisation Error}

Our main results above can be applied to determine the correct normalisation which ensures the discretised stationary GP's will have the same behaviour as the continuous one if we are interested on limit theorems for maximum or its discretised version. Clearly, discrete observations are common in applications, so our findings are directly applicable if particular sampling points or grids are known and observations on those grids are available. Another direction where our results, and in general Piterbarg max-discretisation theorem can be applied is in simulations; again our application concerns the discretisation error and not the simulation error.

Indeed, it is not possible to simulate the maximum (or its standardised version) in continuous time for general GP's, unless the processes are degenerate. A common practice is to simulate the whole processes only on a grid of points, and then use these for calculating the discretised versions of the continuous time maximum. By doing this, we have 
to consider the approximation error due to the calculations based on the discrete grid of points, and the second error is the simulation error. Moreover, since in our case a rare event will be simulated, the simulation error is significant, and can be diminished by using appropriate rare-event simulation techniques, see the recent deep paper Adler et al. (2012).

As it can be seen below, running simulations without going through our analysis is in general not adequate, thus the answer to the question in the Introduction is that running simulations without such analysis as in this paper is not a valid approach.

In order to consider the discretisation error, we need to choose an appropriate grid of points together with an appropriate scaling of the discretised version, and then the rare-event simulation issue needs to be taken care of. Since our novel results above are concerned only with the joint limiting distribution of discrete time and continuous time maximum and its standarised version, they are useful for choosing an appropriate grid of points, and crucial for determining the adequate normalisation for the limit result to hold.

Next, we shall deal with the situation explained by Corollary 2.1. The other results presented above can be utilised in a similar fashion. Consider for simplicity that $\gamma=0$ and the assumptions of the aforementioned corollary hold. First note that for any threshold $u_{T}=x \sqrt{r(T)}+\beta_{T, 0} \widetilde{r}_{T}$ we can approximate the probability that $M_{T}$ exceeds it by $\Phi(x)$. Let us now consider the case of a sparse grid. We have

$$
\begin{aligned}
P\left\{M_{T}>u_{T}\right\}-P\left\{M_{T}^{\delta}>u_{T}\right\} & =P\left\{M_{T}>u_{T}, M_{T}^{\delta} \leq u_{T}\right\} \\
& =P\left\{\frac{M_{T}-\widetilde{r}_{T} \beta_{T, 0}}{r^{1 / 2}(T)}>x, \frac{M_{T}^{\delta}-\widetilde{r}_{T} \beta_{T}^{\delta}}{r^{1 / 2}(T)}+\frac{\widetilde{r}_{T}\left(\beta_{T}^{\delta}-\beta_{T, 0}\right)}{r^{1 / 2}(T)} \leq x\right\} \\
& \rightarrow 1-\Phi(x), \quad T \rightarrow \infty,
\end{aligned}
$$

hence the error of approximating $P\left\{M_{T}>u_{T}\right\}$ by $P\left\{M_{T}^{\delta}>u_{T}\right\}$ is too large, i.e., it does not diminish to 0 as $T \rightarrow \infty$. Consequently, simulating with such grids and using the above approximation is misleading.

Another idea, which is directly motivated by our findings is to approximate $P\left\{M_{T}>u_{T}\right\}$ by $P\left\{M_{T}^{\delta}>u_{T}^{*}\right\}$, with $u_{T}^{*}:=x \sqrt{r(T)}+\beta_{T}^{\delta} \widetilde{r}_{T}$. In this case we have

$$
\begin{aligned}
P\left\{M_{T}>u_{T}\right\}-P\left\{M_{T}^{\delta}>u_{T}^{*}\right\} & =P\left\{M_{T}>u_{T}, M_{T}^{\delta} \leq u_{T}\right\}+P\left\{M_{T}^{\delta}>u_{T}\right\}-P\left\{M_{T}^{\delta}>u_{T}^{*}\right\} \\
& \rightarrow 0, \quad T \rightarrow \infty .
\end{aligned}
$$

Consequently, this approximation is a valid one. Thus if the grid $\mathfrak{R}(\delta)$ is sparse, the right approximation by using the discrete time points is given by our results.

Next, let us assume that the grid of points $\mathfrak{R}(\delta)$ is dense, and instead of computing the survival function of $M_{T}$ we consider the simulation of the survival function of $M_{T}^{\delta}$. We have (which follows also from (15))

$$
P\left\{M_{T}>u_{T}\right\}-P\left\{M_{T}^{\delta}>u_{T}\right\}=P\left\{M_{T}>u_{T}, M_{T}^{\delta} \leq u_{T}\right\} \rightarrow 0, \quad T \rightarrow \infty .
$$

So in the case of a dense grid, we need to use another normalisation of the discretised maximum, again this knowledge is gained by our new result.

Finally, we obtain the constant $c_{T}$ such that the mean error for a dense grid $\mathfrak{R}(\delta)$ satisfies

$$
e_{T}=\mathbb{E}\left\{\frac{M_{T}-M_{T}^{\delta}}{c_{T}}\right\} \rightarrow 0, \quad T \rightarrow \infty .
$$

In the case of dense grid, we know that the convergence

$$
\frac{M_{T}-M_{T}^{\delta}}{b_{T}} \rightarrow 0, \quad T \rightarrow \infty
$$

holds in probability. However the convergence in (16) with $c_{T}=b_{T}$ does not follow automatically. If we impose the following global Hölder condition (see e.g., Theorem D.4 of Piterbarg (1996))

$$
\mathbb{E}\left\{\left(X_{t}-X_{s}\right)^{2}\right\} \leq G|t-s|^{\gamma}, \quad \forall s, t \in(0, \infty)
$$


for some $G>0$ and $\gamma \in(0, \infty)$, then Piterbarg inequality (see Theorem 8.1 of Piterbarg (1996)) holds, i.e.,

$$
P\left\{\sup _{t \in[0, T]}\left|X_{t}\right|>u\right\} \leq C T u^{2 / \gamma-1} \exp \left(-\frac{u^{2}}{2 \sigma_{T}^{2}}\right), \quad \sigma_{T}:=\sup _{t \in[0, T]} \sigma(t)
$$

is valid for any $u$ and $T$ positive with some constant $C>0$ not depending on $u$ and $T$. Piterbarg inequality together with Corollary 2.2 implies in view of Theorem 1 in Seleznjev (2006) (see also Hüsler et al. (2003) for interesting results in this direction) that (16) holds with $c_{T}=b_{T}$.

Summarising we conclude that in view of our findings, we are able to suggest the correct approximation of the continuous time maximum, and similarly for the standardised maximum. The error of approximation for dense grids converges also in the mean to 0 if further the global Hölder condition above holds. The further analysis for bounding the error of the approximation requires a significant effort and is not in the scope of this paper. Similarly, controlling the simulation error requires also additional efforts and cannot be covered here.

Of interest is also to analyse the trade-off between approximation error and simulation error. If the grid is sparse, the approximation error using this discrete grid is larger than when the grid is dense. However simulating on a sparse grid is more efficient and the error can be eventually controlled much easier. Therefore it is not clear without a deep analysis what would be an optimal way to run the simulations, especially since rare-events are to be taken care of, and the time horizon grows with $T$ tending to infinity.

In the recent paper Azaïs and Genz (2012) interesting results are shown for smooth Gaussian processes. Ideas from that paper and Azaïs and Wschbor (2009), Adler et al. (2012) will be important for future research work in the direction of our paper.

\section{Further Results and Proofs}

In this section we present the proofs of our results along with some lemmas which are of some interest on their own. Our notation for asymptotic relations are standard, for instance $f(T) \sim b(T)$ or alternatively $f(T)=g(T)(1+o(1))$ as $T \rightarrow \infty$ mean that the ratio of two real-valued functions $f(T) / g(T)$ tends to 1 as $T \rightarrow \infty$.

We define next the family of random processes index by $T>0$

$$
Y_{T}(t)=\frac{1}{\sigma_{T}(t)}\left(X_{t}-\bar{X}_{T}\right), \quad 0 \leq t \leq T
$$

where $\sigma_{T}^{2}(t)=\mathbb{E}\left\{\left(X_{t}-\bar{X}_{T}\right)^{2}\right\}$. It can be shown as for (2.8) of McCormick (1980) that

$$
\max _{1 \leq t \leq T}\left|\sigma_{T}^{2}(t)-(1-r(T))\right|=o\left(\frac{1}{\ln T}\right)
$$

as $T \rightarrow \infty$. Let $\rho_{T}(s, t)=\mathbb{E}\left\{Y_{T}(s) Y_{T}(t)\right\}$ and $\bar{\rho}_{T}(u)=\max \left\{\left|\rho_{T}(s, t)\right|: 0 \leq s \leq t \leq T, t-s \geq u\right\}$. Again referring to McCormick (1980), see (3.8) therein, we obtain

$$
\max _{0 \leq s, t \leq T}\left|\rho_{T}(s, t)-\frac{r(s-t)-r(T)}{1-r(T)}\right|=o\left(\frac{1}{\ln T}\right) .
$$

By the assumption on $r(t)$, namely $\gamma \in[0,1)$ and (1) for any $\varepsilon>0$ we have $\sup _{t>\varepsilon}|r(t)|<1$, hence, utilising further (18)

$$
\bar{\rho}_{T}(\varepsilon)<1
$$

is valid for all $T$ sufficiently large. Furthermore, we have that for any $\varepsilon>0$ there exists $\tau=\tau(\varepsilon)>0$ such that for all $T$ sufficiently large and $|s-t| \leq \tau$

$$
\frac{1-\varepsilon}{1-\gamma} C|s-t|^{\alpha}+o\left(|s-t|^{\alpha}\right)<1-\rho_{T}(s, t)<\frac{1+\varepsilon}{1-\gamma} C|s-t|^{\alpha}+o\left(|s-t|^{\alpha}\right) .
$$


Define next $M_{T}(Y)=\max _{t \in[0, T]} Y_{T}(t)$ and $M_{T}^{\delta}(Y)=\max _{t \in \mathfrak{R}(\delta) \cap[0, T]} Y_{T}(t)$ and set $\widetilde{r}_{T}:=(1-r(T))^{1 / 2}$. For any $x, y \in \mathbb{R}$, we can further write

$$
\begin{aligned}
& P\left\{a_{T}\left(\Delta_{T}-\beta_{T, \gamma}\right) \leq x, a_{T}\left(\Delta_{T}^{\delta}-\beta_{T}^{*}\right) \leq y\right\} \\
& =P\left\{a_{T}\left(\frac{X_{t}-\bar{X}_{T}}{\sigma_{T}(t)}-\beta_{T, \gamma}\right) \leq x+\vartheta_{T}(t, x), a_{T}\left(\frac{X_{i \delta}-\bar{X}_{T}}{\sigma_{T}(i \delta)}-\beta_{T}^{*}\right) \leq y+\vartheta_{T}^{*}(i \delta, y), t \in[0, T], i \delta \in[0, T]\right\},
\end{aligned}
$$

where

$$
\vartheta_{T}(t, x)=\left(\beta_{T, \gamma} a_{T}+x\right)\left(\frac{\widetilde{r}_{T}}{\sigma_{T}(t)}-1\right), \quad \vartheta_{T}^{*}(i \delta, y)=\left(\beta_{T}^{*} a_{T}+y\right)\left(\frac{\widetilde{r}_{T}}{\sigma_{T}(i \delta)}-1\right)
$$

and $\beta_{T}^{*}=\beta_{T}^{\delta}$ for a sparse grid and $\beta_{T}^{*}=\beta_{T, \gamma}$ for a dense grid. In view of (18)

$$
\max _{0 \leq t \leq T}\left|\vartheta_{T}(t, x)\right|=o(1), \max _{0 \leq i \delta \leq T}\left|\vartheta_{T}^{\delta}(i \delta, y)\right|=o(1) .
$$

Thus, in order to prove (10) and (11), it suffices to show that

$$
\lim _{T \rightarrow \infty} P\left\{a_{T}\left(M_{T}(Y)-\beta_{T, \gamma}\right) \leq x, a_{T}\left(M_{T}^{\delta}(Y)-\beta_{T}^{\delta}\right) \leq y\right\}=\exp (-\exp (-x)-\exp (-y))
$$

and

$$
\lim _{T \rightarrow \infty} P\left\{a_{T}\left(M_{T}(Y)-\beta_{T, \gamma}\right) \leq x, a_{T}\left(M_{T}^{\delta}(Y)-\beta_{T, \gamma}\right) \leq y\right\}=\exp (-\exp (-\min (x, y)))
$$

hold. In order to prove (23) for $\tau=\tau(\varepsilon)$ a fixed constant, we follow a classical scheme, see e.g., Piterbarg (1996). Let therefore $0<a<\tau$ and divide interval $[0, T]$ into intervals of length $\tau-a$ alternating with shorter intervals with length $a$. Denote the long intervals by $S_{k}=[(k-1) \tau+a,(k+1) \tau], k=1,2, \cdots, n=[T / \tau]$, and the short intervals by $R_{k}=((k-1) \tau,(k-1) \tau+a), k=1,2, \cdots, n$. It will be seen from our proofs below that a possible remaining interval with length different than $\tau-a$ or $a$ plays no role in our consideration. We call also this interval a short interval. Denote $\mathbf{S}=\cup S_{k}, \mathbf{R}=\cup R_{k}$, so that $[0, T]=\mathbf{S} \cup \mathbf{R}$. The proof of (23) for the sparse grid case relies on Lemmas 4.1-4.4. Lemma 4.5 will be utilised to prove (24).

Lemma 4.1. For any $B>0$ and for all $x, y \in[-B, B]$ we have

$$
\begin{aligned}
\mid P & \left\{a_{T}\left(M_{T}(Y)-\beta_{T, \gamma}\right) \leq x, a_{T}\left(M_{T}^{\delta}(Y)-\beta_{T}^{\delta}\right) \leq y\right\} \\
& -P\left\{a_{T}\left(\max _{t \in \mathbf{S}} Y_{T}(t)-\beta_{T, \gamma}\right) \leq x, a_{T}\left(\max _{t \in \mathfrak{R}(\delta) \cap \mathbf{S}} Y_{T}(t)-\beta_{T}^{\delta}\right) \leq y\right\} \mid \rightarrow 0
\end{aligned}
$$

as $T \rightarrow \infty$ and $a \downarrow 0$.

Proof: We make use of the following inequality $\left(\right.$ set $u_{T}:=\beta_{T, \gamma}+x / a_{T}, u_{T, \delta}:=\beta_{T}^{\delta}+y / a_{T}$ )

$$
\begin{aligned}
\mid P\{ & \left.a_{T}\left(M_{T}(Y)-\beta_{T, \gamma}\right) \leq x, a_{T}\left(M_{T}^{\delta}(Y)-\beta_{T}^{\delta}\right) \leq y\right\} \\
& -P\left\{a_{T}\left(\max _{t \in \mathbf{S}} Y_{T}(t)-\beta_{T, \gamma}\right) \leq x, a_{T}\left(\max _{t \in \mathfrak{R}(\delta) \cap \mathbf{S}} Y_{T}(t)-\beta_{T}^{\delta}\right) \leq y\right\} \mid \\
& \leq \sum_{k=1}^{n} P\left\{\max _{t \in R_{k}} Y_{T}(t)>u_{T}\right\}+P\left\{\max _{t \in[n \tau+a, T]} Y_{T}(t)>u_{T}\right\} \\
& +\sum_{k=1}^{n} P\left\{\max _{t \in R_{k} \cap \Re(\delta)} Y_{T}(t)>u_{T, \delta}\right\}+P\left\{\max _{k \delta \in[n \tau+a, T]} Y_{T}(k \delta)>u_{T, \delta}\right\}=: I_{1}+I_{2}+I_{3}+I_{4} .
\end{aligned}
$$

Now, let $\xi_{1}(t)$ be a separable standard stationary GP with correlation function $r_{1}(t)$ such that for $|t| \leq \tau$

$$
r_{1}(t)=1-\frac{1+\varepsilon}{1-\gamma} C|t|^{\alpha}+o\left(|t|^{\alpha}\right)
$$


For any $x \in \mathbb{R} u_{T}^{2}=2 \ln T-\ln \ln T+\frac{2}{\alpha} \ln \ln T+O(1)$ as $T \rightarrow \infty$, hence by (21), Slepian Lemma (see Theorem 7.4.2 of Leadbetter et al. (1983)) and Pickands theorem

$$
\begin{aligned}
I_{1}+I_{2} & \leq(n+1) P\left\{\max _{t \in[0, a]} \xi_{1}(t)>u_{T}\right\} \\
& \sim(n+1)\left(\frac{1+\varepsilon}{1-\gamma} C\right)^{1 / \alpha} H_{\alpha} a \varphi\left(u_{T}\right)\left(u_{T}\right)^{2 / \alpha-1} \sim(1+\varepsilon)^{1 / \alpha} \frac{a}{\tau}, \quad T \rightarrow \infty,
\end{aligned}
$$

where $\varphi$ is the density function of the standard Gaussian distribution on $\mathbb{R}$. Note that $\xi_{1}(k \delta), 0 \leq k \delta \leq T$ is a standardized stationary Gaussian random sequence. By (21) and Corollary 4.2.3 of Leadbetter et al. (1983)

$$
\begin{aligned}
I_{3}+I_{4} & \leq(n+1) P\left\{\max _{k \delta \in[0, a]} \xi_{1}(k \delta)>u_{T, \delta}\right\} \\
& \leq(n+1)\left(\left[\frac{a}{\delta}\right]+1\right)\left(1-\Phi\left(u_{T, \delta}\right)\right) \leq \frac{a}{\tau}
\end{aligned}
$$

as $T \rightarrow \infty$. Since $\tau$ is a constant the proof is established by letting $a \downarrow 0$.

Lemma 4.2. Let $q=q_{0}(\ln T)^{-1 / \alpha}$ with $q_{0}>0$. Then

$$
\begin{aligned}
\mid P & \left\{a_{T}\left(\max _{t \in \mathbf{S}} Y_{T}(t)-\beta_{T, \gamma}\right) \leq x, a_{T}\left(\max _{t \in \mathfrak{R}(\delta) \cap \mathbf{S}} Y_{T}(t)-\beta_{T}^{\delta}\right) \leq y\right\} \\
& -P\left\{a_{T}\left(\max _{t \in \mathfrak{R}(q) \cap \mathbf{S}} Y_{T}(t)-\beta_{T, \gamma}\right) \leq x, a_{T}\left(\max _{t \in \mathfrak{R}(\delta) \cap \mathbf{S}} Y_{T}(t)-\beta_{T}^{\delta}\right) \leq y\right\} \mid \rightarrow 0
\end{aligned}
$$

and

$$
\begin{aligned}
\mid & \prod_{k=1}^{n} P\left\{a_{T}\left(\max _{t \in \mathfrak{R}(q) \cap S_{k}} Y_{T}(t)-\beta_{T, \gamma}\right) \leq x, a_{T}\left(\max _{t \in \mathfrak{R}(\delta) \cap S_{k}} Y_{T}(t)-\beta_{T}^{\delta}\right) \leq y\right\} \\
& -\prod_{k=1}^{n} P\left\{a_{T}\left(\max _{t \in S_{k}} Y_{T}(t)-\beta_{T, \gamma}\right) \leq x, a_{T}\left(\max _{t \in \mathfrak{R}(\delta) \cap S_{k}} Y_{T}(t)-\beta_{T}^{\delta}\right) \leq y\right\} \mid \rightarrow 0
\end{aligned}
$$

as $T \rightarrow \infty$ and $q_{0} \downarrow 0$.

Proof: The difference of the two probabilities are bounded above by

$$
\sum_{k=1}^{n}\left(P\left\{a_{T}\left(\max _{t \in \mathfrak{R}(q) \cap S_{k}} Y_{T}(t)-\beta_{T, \gamma}\right) \leq x\right\}-P\left\{a_{T}\left(\max _{t \in S_{k}} Y_{T}(t)-\beta_{T, \gamma}\right) \leq x\right\}\right) .
$$

Let $\xi_{2}(t)$ be a separable standard stationary GP with correlation function $r_{2}(t)$ such that for $|t| \leq \tau$,

$$
r_{2}(t)=1-\frac{1-\varepsilon}{1-\gamma} C|t|^{\alpha}+o\left(|t|^{\alpha}\right)
$$

and let $\xi_{1}(t)$ be defined as before. Applying again Slepian Lemma we have

$$
\begin{aligned}
& \sum_{k=1}^{n}\left(P\left\{a_{T}\left(\max _{t \in \mathfrak{R}(q) \cap S_{k}} Y_{T}(t)-\beta_{T, \gamma}\right) \leq x\right\}-P\left\{a_{T}\left(\max _{t \in S_{k}} Y_{T}(t)-\beta_{T, \gamma}\right) \leq x\right\}\right) \\
& \leq \sum_{k=1}^{n}\left(P\left\{a_{T}\left(\max _{t \in \mathfrak{R}(q) \cap S_{k}} \xi_{2}(t)-\beta_{T, \gamma}\right) \leq x\right\}-P\left\{a_{T}\left(\max _{t \in S_{k}} \xi_{1}(t)-\beta_{T, \gamma}\right) \leq x\right\}\right) \\
& \leq \sum_{k=1}^{n}\left(P\left\{a_{T}\left(\max _{t \in \mathfrak{R}(q) \cap S_{k}} \xi_{2}(t)-\beta_{T, \gamma}\right) \leq x\right\}-P\left\{a_{T}\left(\max _{t \in S_{k}} \xi_{2}(t)-\beta_{T, \gamma}\right) \leq x\right\}\right) \\
& +\sum_{k=1}^{n}\left(P\left\{a_{T}\left(\max _{t \in S_{k}} \xi_{2}(t)-\beta_{T, \gamma}\right) \leq x\right\}-P\left\{a_{T}\left(\max _{t \in S_{k}} \xi_{1}(t)-\beta_{T, \gamma}\right) \leq x\right\}\right)=: J_{1}+J_{2} .
\end{aligned}
$$

Since $\mathfrak{R}(q)$ is a Pickands grid and $\xi_{2}(t)$ is a stationary GP, by Lemma 4 of Piterbarg (2004) we have $J_{1} \rightarrow 0$ as $T \rightarrow \infty$ and $q_{0} \downarrow 0$. By the stationarity of $\xi_{1}(t)$ and $\xi_{2}(t)$ and Pickands theorem again, we obtain

$$
\begin{aligned}
J_{2} & \sim n(\tau-a) C^{1 / \alpha}\left[\left(\frac{1+\varepsilon}{1-\gamma}\right)^{1 / \alpha}-\left(\frac{1-\varepsilon}{1-\gamma}\right)^{1 / \alpha}\right] \varphi\left(x / a_{T}+\beta_{T, \gamma}\right)\left(x / a_{T}+\beta_{T, \gamma}\right)^{2 / \alpha-1} \\
& \sim \frac{\tau-a}{\tau}\left[(1+\varepsilon)^{1 / \alpha}-(1-\varepsilon)^{1 / \alpha}\right] \\
& \rightarrow 0
\end{aligned}
$$


as $T \rightarrow \infty$ and $\varepsilon \downarrow 0$, thus the proof is complete.

Lemma 4.3. Let $q=q_{0}(\ln T)^{-1 / \alpha}$ with $q_{0}>0$. For any $B>0$ and for all $x, y \in[-B, B]$ we have

$$
\begin{aligned}
\lim _{T \rightarrow \infty} \mid P\left\{a_{T}\left(\max _{t \in \mathfrak{R}(q) \cap \mathbf{S}} Y_{T}(t)-\beta_{T, \gamma}\right) \leq x, a_{T}\left(\max _{t \in \mathfrak{R}(\delta) \cap \mathbf{S}} Y_{T}(t)-\beta_{T}^{\delta}\right) \leq y\right\} \\
\quad-\prod_{k=1}^{n} P\left\{a_{T}\left(\max _{t \in \mathfrak{R}(q) \cap S_{k}} Y_{T}(t)-\beta_{T, \gamma}\right) \leq x, a_{T}\left(\max _{t \in \mathfrak{R}(\delta) \cap S_{k}} Y_{T}(t)-\beta_{T}^{\delta}\right) \leq y\right\} \mid=0 .
\end{aligned}
$$

Proof: For the sake of simplicity, let $u_{T}=\beta_{T, \gamma}+x / a_{T}, u_{T, \delta}=\beta_{T}^{\delta}+y / a_{T}$. By Berman's Normal Comparison Lemma (see Theorem 4.2.1 of Leadbetter et al. (1983)) we have with $K$ some constant

$$
\begin{aligned}
& \mid P\left\{a_{T}\left(\max _{t \in \mathfrak{R}(q) \cap \mathbf{S}} Y_{T}(t)-\beta_{T, \gamma}\right) \leq x, a_{T}\left(\max _{t \in \mathfrak{R}(\delta) \cap \mathbf{S}} Y_{T}(t)-\beta_{T}^{\delta}\right) \leq y\right\} \\
& \quad-\prod_{k=1}^{n} P\left\{a_{T}\left(\max _{t \in \mathfrak{R}(q) \cap S_{k}} Y_{T}(t)-\beta_{T, \gamma}\right) \leq x, a_{T}\left(\max _{t \in \mathfrak{R}(\delta) \cap S_{k}} Y_{T}(t)-\beta_{T}^{\delta}\right) \leq y\right\} \mid \\
& \quad \leq K \sum_{s, t \in \mathfrak{R}(q) \cap \mathbf{S},|s-t|>a}\left|\rho_{T}(s, t)\right| \exp \left(-\frac{u_{T}^{2}}{1+\left|\rho_{T}(s, t)\right|}\right) \\
& \quad+K \sum_{s, t \in \mathfrak{R}(\delta) \cap \mathbf{S},|s-t|>a}\left|\rho_{T}(s, t)\right| \exp \left(-\frac{u_{T, \delta}^{2}}{1+\left|\rho_{T}(s, t)\right|}\right) \\
& \quad+K \sum_{s \in \mathfrak{R}(q) \cap \mathbf{S}, t \in \mathfrak{R}(\delta) \cap \mathbf{S},|s-t|>a}\left|\rho_{T}(s, t)\right| \exp \left(-\frac{u_{T}^{2}+u_{T, \delta}^{2}}{2\left(1+\left|\rho_{T}(s, t)\right|\right)}\right) .
\end{aligned}
$$

Following the procedure given on pages 487-489 of McCormick (1980) (recall also (20)), we can prove that each term on the right-hand side of (25) tends to 0 , as $T \rightarrow \infty$, and thus the claim follows.

Lemma 4.4. For the $G P\left\{Y_{T}(t), t \in[0, T]\right\}$ and for any $x, y \in \mathbb{R}$ we have

$$
\left|\prod_{k=1}^{n} P\left\{a_{T}\left(\max _{t \in S_{k}} Y_{T}(t)-\beta_{T, \gamma}\right) \leq x, a_{T}\left(\max _{t \in \mathfrak{R}(\delta) \cap S_{k}} Y_{T}(t)-\beta_{T}^{\delta}\right) \leq y\right\}-\exp (-\exp (-x)-\exp (-y))\right| \rightarrow 0
$$

as $T \rightarrow \infty$ and $\varepsilon \downarrow 0$.

Proof: By (21) and Slepian Lemma

$$
\begin{aligned}
& \left(P\left\{a_{T}\left(\max _{t \in S_{1}} \xi_{1}(t)-\beta_{T, \gamma}\right) \leq x, a_{T}\left(\max _{t \in \mathfrak{R}(\delta) \cap S_{1}} \xi_{1}(t)-\beta_{T}^{\delta}\right) \leq y\right\}\right)^{n} \\
& \leq \prod_{k=1}^{n} P\left\{a_{T}\left(\max _{t \in S_{k}} Y_{T}(t)-\beta_{T, \gamma}\right) \leq x, a_{T}\left(\max _{t \in \mathfrak{R}(\delta) \cap S_{k}} Y_{T}(t)-\beta_{T}^{\delta}\right) \leq y\right\} \\
& \leq\left(P\left\{a_{T}\left(\max _{t \in S_{1}} \xi_{2}(t)-\beta_{T, \gamma}\right) \leq x, a_{T}\left(\max _{t \in \mathfrak{R}(\delta) \cap S_{1}} \xi_{2}(t)-\beta_{T}^{\delta}\right) \leq y\right\}\right)^{n} .
\end{aligned}
$$

Thus, we can derive an upper bound as

$$
\begin{aligned}
& \prod_{k=1}^{n} P\left\{a_{T}\left(\max _{t \in S_{k}} Y_{T}(t)-\beta_{T, \gamma}\right) \leq x, a_{T}\left(\max _{t \in \mathfrak{R}(\delta) \cap S_{k}} Y_{T}(t)-\beta_{T}^{\delta}\right) \leq y\right\} \\
& \leq \exp \left(n \ln \left(P\left\{a_{T}\left(\max _{t \in S_{1}} \xi_{2}(t)-\beta_{T, \gamma}\right) \leq x, a_{T}\left(\max _{t \in \mathfrak{R}(\delta) \cap S_{1}} \xi_{2}(t)-\beta_{T}^{\delta}\right) \leq y\right\}\right)\right) \\
& =\exp \left(-n\left(1-P\left\{a_{T}\left(\max _{t \in S_{1}} \xi_{2}(t)-\beta_{T, \gamma}\right) \leq x, a_{T}\left(\max _{t \in \mathfrak{R}(\delta) \cap S_{1}} \xi_{2}(t)-\beta_{T}^{\delta}\right) \leq y\right\}\right)+R_{n}\right) .
\end{aligned}
$$

Since

$$
P_{n}=: P\left\{a_{T}\left(\max _{t \in S_{1}} \xi_{2}(t)-\beta_{T, \gamma}\right) \leq x, a_{T}\left(\max _{t \in \mathfrak{R}(\delta) \cap S_{1}} \xi_{2}(t)-\beta_{T}^{\delta}\right) \leq y\right\} \rightarrow 1, \quad T \rightarrow \infty,
$$


then $R_{n}=o\left(n\left(1-P_{n}\right)\right)$ as $T \rightarrow \infty$. Now, applying Lemma 2 of Piterbarg (2004) we obtain

$$
\begin{aligned}
& n\left(1-P\left\{a_{T}\left(\max _{t \in S_{1}} \xi_{2}(t)-\beta_{T, \gamma}\right) \leq x, a_{T}\left(\max _{t \in \mathfrak{R}(\delta) \cap S_{1}} \xi_{2}(t)-\beta_{T}^{\delta}\right) \leq y\right\}\right) \\
& \sim n\left(P\left\{a_{T}\left(\max _{t \in S_{1}} \xi_{2}(t)-\beta_{T, \gamma}\right)>x\right\}+P\left\{a_{T}\left(\max _{t \in \mathfrak{R}(\delta) \cap S_{1}} \xi_{2}(t)-\beta_{T}^{\delta}\right)>y\right\}\right) \\
& \sim n(\tau-a) T^{-1}(1-\varepsilon)^{1 / \alpha} e^{-x}+n(\tau-a) T^{-1} e^{-y} \\
& \sim(1-\varepsilon)^{1 / \alpha} e^{-x}+e^{-y}, \quad T \rightarrow \infty .
\end{aligned}
$$

Consequently

$$
\prod_{k=1}^{n} P\left\{a_{T}\left(\max _{t \in S_{k}} Y_{T}(t)-\beta_{T, \gamma}\right) \leq x, a_{T}\left(\max _{t \in \mathfrak{R}(\delta) \cap S_{k}} Y_{T}(t)-\beta_{T}^{\delta}\right) \leq y\right\} \leq \exp \left(-\left((1-\varepsilon)^{1 / \alpha} e^{-x}+e^{-y}\right)\right) .
$$

A similar argument leads to the lower bound

$$
\prod_{k=1}^{n} P\left\{a_{T}\left(\max _{t \in S_{k}} Y_{T}(t)-\beta_{T, \gamma}\right) \leq x, a_{T}\left(\max _{t \in \mathfrak{R}(\delta) \cap S_{k}} Y_{T}(t)-\beta_{T}^{\delta}\right) \leq y\right\} \geq \exp \left(-\left((1+\varepsilon)^{1 / \alpha} e^{-x}+e^{-y}\right)\right),
$$

hence letting $\varepsilon \downarrow 0$ establishes the proof.

Lemma 4.5. For any dense grid $\mathfrak{R}(\delta)$ and any $x \in \mathbb{R}$

$$
\lim _{T \rightarrow \infty}\left|P\left\{a_{T}\left(\max _{t \in[0, T]} Y_{T}(t)-\beta_{T, \gamma}\right) \leq x\right\}-P\left\{a_{T}\left(\max _{t \in \mathfrak{R}(\delta) \cap[0, T]} Y_{T}(t)-\beta_{T, \gamma}\right) \leq x\right\}\right|=0 .
$$

Proof: For any $x \in \mathbb{R}$

$$
\begin{aligned}
& \left|P\left\{a_{T}\left(\max _{t \in[0, T]} Y_{T}(t)-\beta_{T, \gamma}\right) \leq x\right\}-P\left\{a_{T}\left(\max _{t \in \mathfrak{R}(\delta) \cap[0, T]} Y_{T}(t)-\beta_{T, \gamma}\right) \leq x\right\}\right| \\
& \leq\left|P\left\{a_{T}\left(\max _{t \in[0, T]} Y_{T}(t)-\beta_{T, \gamma}\right) \leq x\right\}-P\left\{a_{T}\left(\max _{t \in \mathbf{S}} Y_{T}(t)-\beta_{T, \gamma}\right) \leq x\right\}\right| \\
& +\left|P\left\{a_{T}\left(\max _{t \in \mathbf{S}} Y_{T}(t)-\beta_{T, \gamma}\right) \leq x\right\}-P\left\{a_{T}\left(\max _{t \in \mathfrak{R}(\delta) \cap \mathbf{S}} Y_{T}(t)-\beta_{T, \gamma}\right) \leq x\right\}\right| \\
& +\left|P\left\{a_{T}\left(\max _{t \in \mathfrak{R}(\delta) \cap \mathbf{S}} Y_{T}(t)-\beta_{T, \gamma}\right) \leq x\right\}-P\left\{a_{T}\left(\max _{t \in \mathfrak{R}(\delta) \cap[0, T]} Y_{T}(t)-\beta_{T, \gamma}\right) \leq x\right\}\right|=: L_{1}+L_{2}+L_{3} .
\end{aligned}
$$

Utilising a similar argument as in the proof of Lemma 4.1, we get

$$
\lim _{T \rightarrow \infty} L_{1}=\lim _{T \rightarrow \infty} L_{3}=0 .
$$

Note that $\mathfrak{R}(\delta)$ is a dense grid. Form the proof of Lemma 4.2, we know that $\lim _{T \rightarrow \infty} L_{2}=0$, hence the proof is complete.

Proof of Theorem 1.1. The first claim follows directly by Lemmas 4.1-4.4. We will prove the asymptotic relation (24). In view of Lemma 4.5 we have

$$
\begin{aligned}
& \left|P\left\{a_{T}\left(M_{T}(Y)-\beta_{T, \gamma}\right) \leq x, a_{T}\left(M_{T}^{\delta}(Y)-\beta_{T, \gamma}\right) \leq y\right\}-P\left\{a_{T}\left(M_{T}(Y)-\beta_{T, \gamma}\right) \leq x, a_{T}\left(M_{T}(Y)-\beta_{T, \gamma}\right) \leq y\right\}\right| \\
& \leq\left|P\left\{a_{T}\left(M_{T}^{\delta}(Y)-\beta_{T, \gamma}\right) \leq y\right\}-P\left\{a_{T}\left(M_{T}(Y)-\beta_{T, \gamma}\right) \leq y\right\}\right| \rightarrow 0, \quad T \rightarrow \infty .
\end{aligned}
$$

Next, applying (22) and Theorem A

$$
\begin{aligned}
P\left\{a_{T}\left(M_{T}(Y)-\beta_{T, \gamma}\right) \leq x, a_{T}\left(M_{T}(Y)-\beta_{T, \gamma}\right) \leq y\right\} & =P\left\{a_{T}\left(M_{T}(Y)-\beta_{T, \gamma}\right) \leq \min (x, y)\right\} \\
& \sim P\left\{a_{T}\left(\Delta_{T}-\beta_{T, \gamma}\right) \leq \min (x, y)\right\} \\
& \rightarrow \exp (-\exp (-\min (x, y)))
\end{aligned}
$$


holds as $T \rightarrow \infty$, and thus the proof is complete.

Proof of Corollary 2.1. Note first that $\left(\right.$ set $\left.\widetilde{r}_{T}=(1-r(T))^{1 / 2}\right)$

$$
r^{-1 / 2}(T)\left(\frac{M_{T}}{\widetilde{r}_{T}}-\beta_{T, \gamma}\right)=\left(r^{1 / 2}(T) a_{T}\right)^{-1} a_{T}\left[\Delta_{T}-\beta_{T, \gamma}\right]+r^{-1 / 2}(T) \bar{X}_{T} / \widetilde{r}_{T}
$$

and

$$
r^{-1 / 2}(T)\left(\frac{M_{T}^{\delta}}{\widetilde{r}_{T}}-\beta_{T}^{*}\right)=\left(r^{1 / 2}(T) a_{T}\right)^{-1} a_{T}\left[\Delta_{T}^{\delta}-\beta_{T}^{*}\right]+r^{-1 / 2}(T) \bar{X}_{T} / \widetilde{r}_{T}
$$

Both first terms in the above two sums convergence in probability to 0 by Theorem 1.1 and the assumption $(r(T) \ln T)^{-1}=o(1)$. Consequently, utilising further the fact that the variance of the second term tends to $(1-\gamma)^{-1}$ as $T \rightarrow \infty$, we have

$$
\begin{aligned}
& P\left\{r^{-1 / 2}(T)\left(\frac{M_{T}}{\widetilde{r}_{T}}-\beta_{T, \gamma}\right) \leq x, r^{-1 / 2}(T)\left(\frac{M_{T}^{\delta}}{\widetilde{r}_{T}}-\beta_{T}^{*}\right) \leq y\right\} \\
& \sim P\left\{r^{-1 / 2}(T) \bar{X}_{T} / \widetilde{r}_{T} \leq x, r^{-1 / 2}(T) \bar{X}_{T} / \widetilde{r}_{T} \leq y\right\}=P\left\{r^{-1 / 2}(T) \bar{X}_{T} / \widetilde{r}_{T} \leq \min (x, y)\right\} \\
& \rightarrow\left(\frac{1-\gamma}{2 \pi}\right)^{1 / 2} \int_{-\infty}^{\min (x, y)} \exp \left(-\frac{1-\gamma}{2} z^{2}\right) d z, \quad T \rightarrow \infty,
\end{aligned}
$$

hence the claim follows.

Acknowledgement: We thank both referees and the Editor-in-Chief for several important suggestions and corrections. Both authors acknowledge partial support by the Swiss National Science Foundation Grant 200021-1401633/1.

\section{References}

[1] Abramowicz, K., Seleznjev, O., Multivariate piecewise linear interpolation of a random field. arXiv:1102.1871, 2011a.

[2] Abramowicz, K., Seleznjev, O., Spline approximation of a random process with singularity. J. Stat. Plan. Inf. 2011b, 141, 1333-1342.

[3] Abramowicz, K., Seleznjev, O., Stratified Monte Carlo quadrature for continuous random fields. arXiv:1104.4920, 2011c.

[4] Adler, R.J., Blanchet, J., Liu, J., Efficient monte carlo for high excursions of Gaussian random fields. Ann. Appl. Probab. 2012, 1167-1214.

[5] Albin, J.M.P., Choi, A., A new proof of an old result by Pickands, Electron. Commun. Probab., 2010, 15, 339-345.

[6] Azaïs, J.-M., Genz, A., Computation of the distribution of the maximum of stationary Gaussian processes. Meth. Computing Appl. Probab. 2012, (in press).

[7] Azaïs, J.-M., Wschbor, M., Level sets and extrema of random processes and fields. Wiley, Hoboken, N.J., 2009.

[8] Berman, S.M., Limiting distribution of the studentized largest observation. Skand. Akt., 1962, 45, $154-161$.

[9] Berman, S.M., Sojourns and extremes of Gaussian processes. Ann. Probab., 1974, 2, 999-1026.

[10] Grubbs, F.E., Sample criteria for testing outlying observations. Ann. Math. Statist., 1964, 35, 512-516.

[11] Dębicki, K., Ruin probability for Gaussian integrated processes. Stochastic Process. Appl., 2002, 98, 151-174.

[12] Dȩbicki, K., Kisowski, P., A note on upper estimates for Pickands constants. Stat. Prob. Letters, 2009, 78, $2046-2051$.

[13] Dębicki, K., Tabiś, K., Extremes of time-average stationary Gaussian processes. Stochastic Process. Appl., 2011, 121, 2049-2063. 
[14] Falk, M., Hüsler, J., Reiss, R.-D., Laws of Small Numbers: Extremes and Rare Events. DMV Seminar Vol. 23, 2nd edn., Birkhäuser, Basel, 2010.

[15] Ho, H.C., McCormick, W.P., Asymptotic distribution of sum and maximum for Gaussian processes. J. Appl. Probab., 1999, 36, 1031-1044.

[16] Hashorva, E., Hüsler, J., Extremes of Gaussian processes with maximal variance near the boundary points. Meth. Computing Appl. Probab., 2000, 2, 255-269.

[17] Hashorva, E., Lifshits, M.A., Seleznjev, O., Approximation of a random process with variable smoothness. arXiv:1206.1251v1, 2012.

[18] Hüsler, J., Extreme values and high boundary crossings for locally stationary Gaussian processes. Ann. Probab., 1990, 18, 1141-1158.

[19] Hüsler, J., Dependence between extreme values of discrete and continuous time locally stationary Gaussian processes. Extremes, 2004, 7, 179-190.

[20] Hüsler, J., Piterbarg, V.I., Seleznjev, O., On convergence of the uniform norms for Gaussian processes and linear approximation problems. Ann. Appl. Probab. 2003, 13, 1615-1653.

[21] Hüsler, J., Piterbarg, V., Limit theorem for maximum of the storage process with fractional Brownian motion as input. Stoch. Process. Appl., 2004, 114, 231-250.

[22] James, B., James, K., Qi, Y., Limit distribution of the sum and maximum from multivariate Gaussian sequences. J. Multi. Analysis, 2007, 98, 517-532

[23] Kabluchko, Z., Extremes of independent Gaussian processes. Extremes, 2011, 14, 285-310.

[24] Lifshits, M.A., Gaussian Random Functions. Kluwer, Dordrecht, 1995.

[25] Leadbetter, M.R., Lindgren, G., Rootzén, H., Extremes and Related Properties of Random Sequences and Processes. Series in Statistics. Springer, New York, 1983.

[26] McCormick, W.P., Weak convergence for the maxima of stationary Gaussian processes using random normalization. Ann. Probab., 1980, 8, 498-510.

[27] McCormick, W.P., Qi, Y., Asymptotic distribution for the sum and maxima of Gaussian processes. J. Appl. Probab., $2000,37,958-971$.

[28] Mittal, Y., Ylvisaker, D., Limit distribution for the maximum of stationary Gaussian processes. Stoch. Process. Appl., $1975,3,1-18$.

[29] Oesting, M., Kabluchko, Z. and Schlather, M., Simulation of BrownResnick processes. Extremes, 2012, $15,89-107$.

[30] Pickands, J.III., Asymptotic properties of the maximum in a stationary Gaussian process. Trans. Am. Math. Soc., 1969, $145,75-86$

[31] Piterbarg, V.I., On the paper by J. Pickands "Upcrosssing probabilities for stationary Gaussian processes". Vestnik Moscow. Univ. Ser. I Mat. Mekh. 27, 25-30. English translation in Moscow Univ. Math. Bull., $1972,27$.

[32] Piterbarg, V.I., Discrete and continuous time extremes of Gaussian processes. Extremes, 2004, 7, $161-177$.

[33] Piterbarg, V.I., Asymptotic Methods in the Theory of Gaussian Processes and Fields. AMS, Providence, 1996.

[34] Seleznjev, O., Asymptotic behavior of mean uniform norms for sequences of Gaussian processes and fields. Extremes, 2006, 8, 161-169. 
[35] Tan, Z., Wang, Y., Extreme values of discrete and continuous time strongly dependent Gaussian processes. Comm. Stat. Theory Meth., 2012, in press.

[36] Turkman, K.F., Discrete and Continuous time series extremes of stationary processes. Handbook of statistics Vol 30. Time Series Methods and Aplications. Eds. T.S. Rao, S.S. Rao and C.R. Rao. Elsevier, pp. 565-580, 2012. 